C2008 IEEE. Personal use of this material is permitted. However, permission to reprint/republish this material for advertising or promotional purposes or for creating new collective works for resale or redistribution to servers or lists, or to reuse any copyrighted component of this work in other works must be obtained from the IEEE. 


\title{
Towards the Mental Health Ontology
}

\author{
Maja Hadzic, Meifania Chen, Tharam S. Dillon \\ Research Lab for Digital Health Ecosystems, Curtin University of Technology, Perth, Australia \\ m.hadzic@curtin.edu.au,m.chen@curtin.edu.au, t.dillon@curtin.edu.au
}

\begin{abstract}
Lots of research have been done within the mental health domain, but exact causes of mental illness are still unknown. Concerningly, the number of people being affected by mental conditions is rapidly increasing and it has been predicted that depression would be the world's leading cause of disability by 2020 . Most mental health information is found in electronic form. Application of the cutting-edge information technologies within the mental health domain has the potential to greatly increase the value of the available information. Specifically, ontologies form the basis for collaboration between research teams, for creation of semantic web services and intelligent multi-agent systems, for intelligent information retrieval, and for automatic data analysis such as data mining. In this paper, we present Mental Health Ontology which can be used to underpin a variety of automatic tasks and positively transform the way information is being managed and used within the mental health domain.
\end{abstract}

\section{INTRODUCTION}

Mental illness is becoming one of the major problems of our society [1]. The World Health Organization predicted that depression would be the world's leading cause of disability by 2020 [2]. It has also been proven that mental illness is a causal factor in many chronic conditions such as diabetes, hypertension, HIV/AIDS resulting in higher cost to the health system [3].

An ontology provides a shared common understanding of a domain. As ontologies are highly expressive knowledge models they can be used to increase the expressiveness and intelligence of a system [4,5]. An ontology, particularly in medicine, grew out of a perceived need for a controlled vocabulary [6,7]. The importance of ontologies has been recognised within the biomedical domain and work has begun on developing and sharing biomedical ontologies [8,9] such as Gene Ontology (GO) (http://www.geneontology.org/), Unified Medical Language System (UMLS) [10], Human Disease Ontology [11], Protein Ontology (http://proteinontology.info/) [12], etc. A great variety of biomedical ontologies is available via The Open Biomedical Ontologies (http://obofoundry.org/) covering various domains such as anatomy, biological processes, biochemistry, health and taxonomy.

We have identified five main application areas for ontologies:

1. Ontology-based Collaboration Between Research Teams

2. Ontology-based Web Services

3. Ontology-mediated Information Access and Retrieval

4. Ontology-based Multi-agent Systems

\section{Ontology-aided Data Mining}

Ontologies are used in situations where collaboration between research teams needs to be established. For example, research in mental illness requires collaboration and sharing of information as different research teams may work on different aspects of mental illness. Identification of the precise patterns of causal factors responsible for a specific type of mental illness still remains unsolved and is therefore a very active research focus today $[13,14,15,16,17]$. Some researchers suggest that a bacterial or viral infection causes mental illness [17].

The importance of ontologies within the Web Services community is great as ontologies can provide a semantic framework that will enable knowledge sharing and use by all parties involved. In some of our works $[18,19]$ we have explained how Ontology technology in combination with Grid technology can help create collaborative environments and increase interoperability within the system.

Information regarding mental illness is dispersed over various resources and it is difficult to link this information, to share it and find specific information when needed. A Mental Health Ontology can be designed to provide a model of mental health concepts and relationships that can be used to form a semantic framework for many data storage and retrieval. Such a semantic framework could be used for systematic annotation of mental health information available through various information resources and support querying of heterogeneous information sources.

A collection of agents can utilise a shared domain ontology as their common knowledge base. This will facilitate communication and coordination between agents and support some important processes within a multi-agent system such as: problem decomposition and task sharing among different agents, results sharing and analysis, information retrieval, selection and integration etc [20].

A Mental Health Ontology can form a semantic framework for the data mining tasks which can play a crucial role in deriving knowledge and assisting in the prevention, diagnosis, treatment and control of mental illness. Data mining algorithms have great potential to expose the patterns in mental health data, facilitate the search for the combinations of genetic and environmental factors involved and provide an indication of influence [21, 22].

\section{Mental Health Ontology Model}

The Mental Health Ontology (MHO) consists of three subontologies which represent (1) disorder types, (2) factors and (3) treatments. 


\section{A. Disorder Types}

There are currently two established classification systems for mental health disorders which list categories of disorder types, namely the International Statistical Classification of Diseases and Related Health Problems, $10^{\text {th }}$ Revision (ICD10) published by the World Health Organization (WHO) [23] and the Diagnostic and Statistical Manual of Mental Disorders, Fourth Revision (DSM-IV) by the American Psychiatry Association [24]. Both classification systems are essentially congruent in content due to internationally available knowledge of mental disorders in the literature, although some differences still remain. A key disparity between the two is their origins and consequently, target groups. The DSM is predominantly based on research studies conducted in the United States, as a result excluding concepts of disorders prevalent in other cultures [25]. On the other hand, the ICD-10 is an international standard diagnostic classification of a wide range of health conditions, among which chapter $\mathrm{V}$ is dedicated to "mental and behavioral disorders". As such, diagnoses based on the ICD-10 show a high reliability between different institutions and a good international comparability [26]. Another notable difference between the two classification systems is the content. The WHO states that the term "mental disorder" can include mental retardation in addition to mental illness, personality disorders and substance dependence [27]. In contrast, mental retardation is not classified as a clinical disorder in DSM-IV [24]. Despite their differences, both ICD-10 and DSM-IV classification systems have achieved worldwide acceptance. A survey of 205 psychiatrists, from 66 different countries across all continents, reported that ICD-10 was more frequently used in clinical practice and training, while the DSM-IV was more valued for research [28].

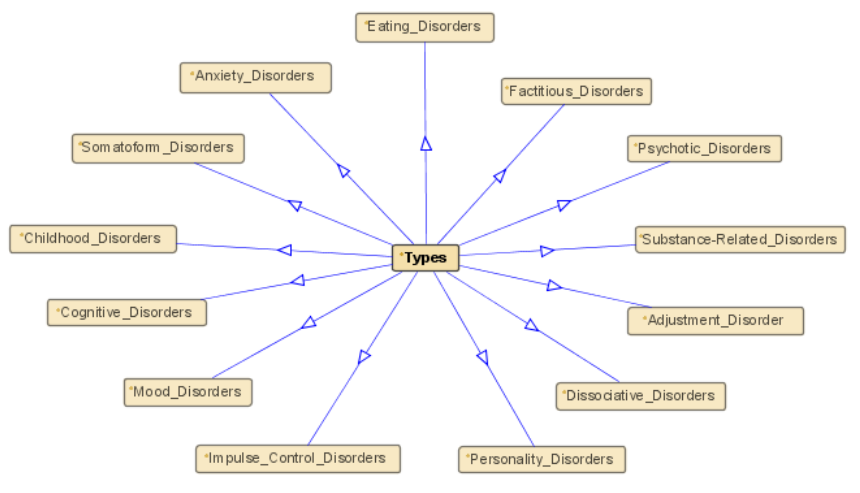

Fig. 1. Ontology model of the Types sub-ontology (sub-classes not shown).

By collating information from the two classification schemes that best represents mental health disorders, we have identified 13 types of mental illnesses along with their individual sub-classes, shown in Fig. 1 and Fig. 2 respectively. Mental retardation was not included in the MHO since our focus is on clinical disorders. The WHO has recently conducted an international survey of mental disorders across 26 countries based on ICD-10 and DSM-IV criteria. Based on the first published results from 14 countries completed to date, anxiety disorders were found to be the most prevalent in all but one country (prevalence in a 12month period of $2.4 \%$ to $18.2 \%$ ), followed by mood disorders which are most common in all but two countries $(0.8 \%$ to 9.6\%), while substance disorders $(0.1 \%-6.4 \%)$ and impulsecontrol disorders $(0.0 \%-6.8 \%)$ were less prevalent [29]. However, these are believed to be underestimated, due to poor diagnosis, especially in countries without affordable access to mental health services, and low reporting rates, as a consequence of the predominant use of self-report data.

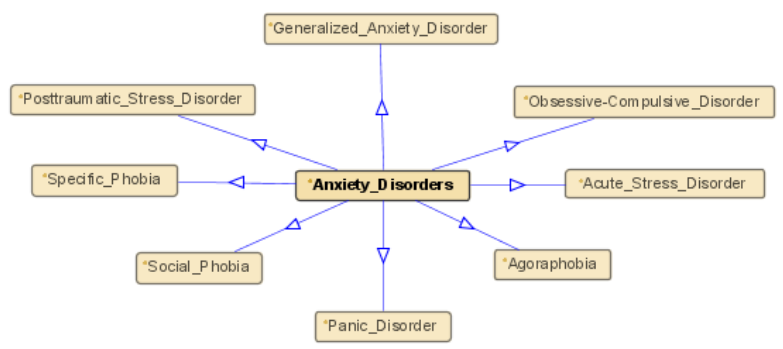

Fig. 2. An example of further sub-ontology classification, i.e. further classification of anxiety disorder from the Types sub-ontology.

\section{B. Factors (causes)}

As we have explained in Section II, the exact causes of mental illness are still unknown. Some causal factors such as genetic, environmental and microbial, have been identified but their individual influences on mental health are still unknown. One of the sub-ontologies of MHO has been designed to capture and represent the knowledge related to the factors affecting the mental health of individuals. We have classified those factors under the following 5 categories: (1) genetic, (2) physical, (3) environmental, (4) personal and (5) microorganisms.

Genetic factors include variations/mutations of human DNA that affect mental health. Our model will capture information about 'genes', 'gene complexes' (such as G72/G30 gene complex associated with Schizophrenia [42]) and 'DNA regions of interest' i.e. regions in DNA that potentially contain mutated genes affecting mental health.

Physical factors define and describe physical conditions that may affect mental health. These include 'Addison's disease', 'Acute Porphyria', 'Cushing's syndrome', 'Corticosteroid treatment', 'Cerebral abscess', 'Cerebrovascular accident', 'Cerebral tumours', 'Encephalitis', 'Encephalitis lethargica', 'Epilepsy', 'Head injury', 'Hyperparathyroidism', 'Hyperthyroidism', 'Hypothyroidism', 'Hypoparathyroidism', 'Hypopituitarism', 'Huntington's chorea', 'Insulinomas', 'Liver disease', 'Multiple sclerosis', 'Neurosyphilis', 'Subarachnoid 


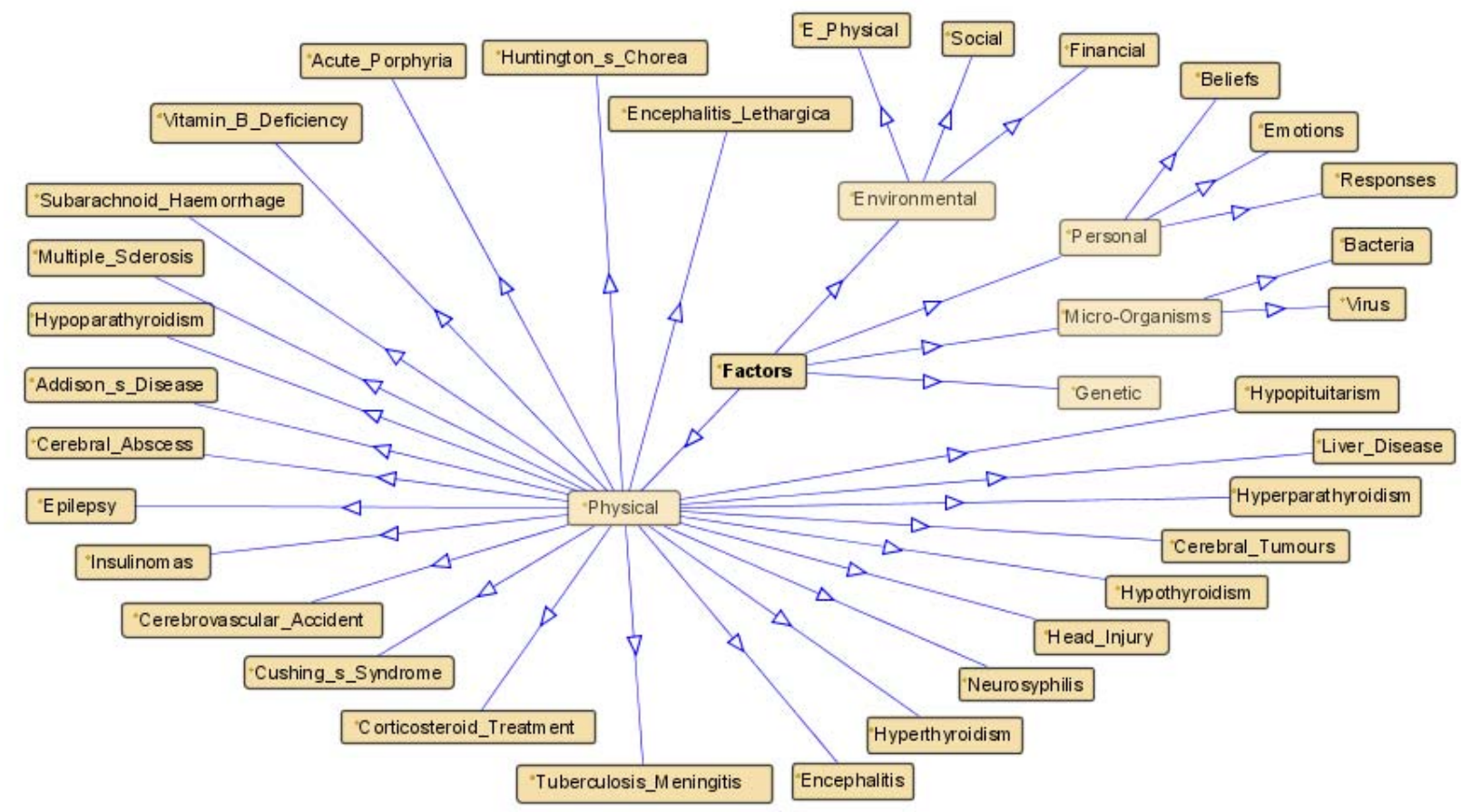

Fig. 3. Ontology model of factors that contribute to mental health disorders. Sub-ontologies include physical, environmental, personal, micro-organisms and genetic factors, and are further divided into their individual sub-types.

haemorrhage', 'Tuberculosis meningitis', 'Vitamin B deficiency', etc. For example, Vitamin B deficiency may result in depression, liver failure may cause hallucinations, multiple sclerosis may result in mood disorders, and Tuberculosis meningitis may result in personality disorders. The relationship between physical and mental health has also been reported by Tsevat et al. [43].

Environmental factors include factors surrounding us that we have less control of. For example, our 'physical environment' is determined by 'climate', 'living conditions', 'noise', 'pollution', etc. A large number of people are affected by weather. Usually, overcast weather affects people negatively and sunny weather positively, while noise can increase stress levels and affect mental health in a negative way. 'Social environment' captures factors determined by our relationships with others and includes both damaging relationships such as 'conflicts', 'abuse' and 'discrimination' as well as beneficial relationships. It has also been reported that the 'Financial environment' affects our health. Ettner [44] provides evidence that increases in income significantly improve physical and mental health.

Personal factors relate to the factors surrounding us that we have more control over. It has been reported [45] that what we 'believe' in and our spiritual levels affect our mental health. D'Souza $[46,47]$ highlights the need of patients to have their spiritual issues addressed. Our immediate 'responses' regarding complex situations can have a longterm impact on our mental health. These responses can be directed 'towards ourselves' or 'towards others'. Negative circumstances can inspire some to 'problem solving', while others may tend towards 'isolation' or 'drug abuse'. Some may be inspired to 'forgiveness', others will put 'blame' on someone. One of the greatest effects on our mental health, and health in general is our 'emotions' [48]. Some of these include 'stress', 'anger', 'bitterness', 'guilt', 'joy', 'happiness', 'peace', 'fear', and are directly affecting our mental health.

Recent news has reported possible existence of microorganisms such as 'virus' or 'bacteria' that may affect mental health. More research is required to explain why mental illness appears to be transmittable; is this caused by a microorganism or is the wellness/illness 'contiguous'?

\section{Treatments}

There are various ways to manage mental health disorders. Treatments include pharmacotherapy, psychotherapy, group and family therapy, electroconvulsive therapy and psychosurgery. A number of studies have established the correlation between chemical imbalances in the brain and specific psychiatric conditions which subsequently led to the development of pharmacotherapy $[30,31]$. These psychoactive drugs are categorized according to the disorder for which they are primarily prescribed, and are a highly effective treatment for the targeted disorder. In recent years, significant advances have been made in the field of psychotherapy, an interpersonal intervention which employs one or more of a range of specific psychological techniques facilitated through a psychotherapist. These include behavioural therapy [32, 33], cognitive therapy [34, 35], 


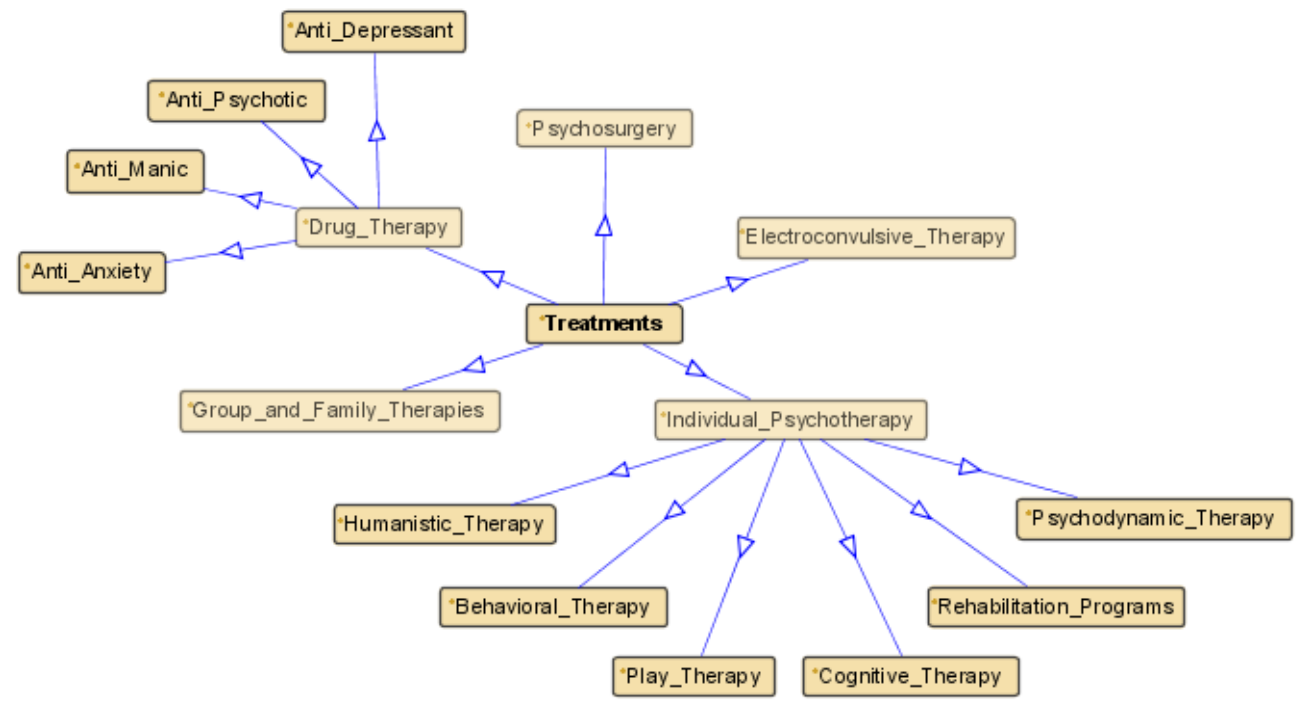

Fig. 4. Ontology model of treatments for mental health disorders. Sub-ontologies include drug therapy, psychosurgery, electroconvulsive therapy, individual psychotherapy, and group and family therapies, and are further divided into their individual sub-types.

humanistic therapy [36], play therapy [37], psychodynamic therapy $[38,39]$ as well as rehabilitation programs. Group and family therapies are also often useful in coping with stress. Most studies suggest that an integrated treatment approach involving both drugs and psychotherapy is more effective than either treatment method used alone [40, 41].

\section{CONCLUSION}

The three ontology 'dimensions' (illness type, factors and treatments) contain very different information and are orthogonal to each other. The 'Illness Types' sub-ontology is more a classifying ontology and is strongly hierarchically supported. The 'Factors' sub-ontology is strongly based on scientific research and expose different kind of factors that may affect our mental health, both positively and negatively. The 'Treatment' sub-ontology is a combination of classifying and research ontology. Designing new drugs is research work but, for example, all the discovered drugs can be hierarchically classified. All three 'dimensions' are different from each other and each 'dimension' is unique. But jointly they give an overall picture and a good overview of mental health knowledge.

The mental health domain is a very complex discipline, and requires breaking the information silos and conducting crossdisciplinary research. Mental illness is not simply a case of blood tests and prescription of medications. It is much more than that. There is a need for physiologists, molecular biologists, biochemists, neurologists, neuroscientists, psychologists, psychiatrists, drug therapists, herbalists, sociologists, theologises, etc. as well as information and computer scientists to come together and build intelligent systems to aid medical researchers, physicians, patients and general public in efficient and effective use, management and retrieval of mental health information. Such systems go some way to delivering what Patel et al [49] say is 'necessary to transform the quality of mental health care'. They improve the infrastructure for evidence-based interventions and provide innovation for quality improvement in mental health care.

Of specific importance is information about factors affecting mental health. As this is still a grey area and exact causes of mental disorders are unclear, precise treatment strategies cannot be developed at this stage. The doctors are often forced to prescribe medication which may give temporal relief but in reality mask the real issue and often result in side effects that will make the patient's situation even worse. Medical researchers need support in advancing their research in identifying the illness causing factors and effective patient treatments. This would reduce the possibility of redundant research (saving research time, effort and resources) and facilitate development of technologies for maintaining good health. The general public should also have access to accurate, reliable and up-to-date information that will help their understanding of mental health and motivate them to control their mental health better. Additionally, reducing the number of mentally ill patients will significantly reduce the cost of the mental health budget.

In our research centre, we are developing MHO using Protégé. We hope this is only the beginning of our future mental heath projects which will incorporate agent-based systems and data mining. We are inviting other researchers that share the same vision to join us in these projects.

\section{REFERENCES}

[1] Australian Government, "Department of Health and Ageing Factbook 2006",http://www.health.gov.au/internet/wcms/publishing.nsf/Content/ Factbook2006-1 (accessed 20 February 2007)

[2] A.D. Lopez, C.C.J.L. Murray, "The global burden of disease, 19902020”, Nature Medicine, vol. 4, pp. 1241-1243, 1998. 
[3] M. Horvitz-Lennon, A.M. Kilbourne, H.A. Pincus, "From silos to bridges: meeting the general health care needs of adults with severe mental illnesses", Health Affairs, vol. 25, no. 3, pp. 659-669, 2006.

[4] A. Gómez-Pérez, "Towards a framework to verify knowledge sharing technology", Expert Systems with Applications, vol.11, no.4, pp.519529, 1996.

[5] A. Gómez-Pérez, "Knowledge sharing and reuse", The Handbook on Applied Expert Systems, CRC Press, 1998, pp. 1-36.

[6] J.J. Cimino, "In defense of the desiderata", Journal of Biomedical Informatics, vol.39, pp.299-306, 2006.

[7] B. Smith, "From concepts to clinical reality: An essay on the benchmarking of biomedical terminologies", Journal of Biomedical Informatics, vol.39, pp.288-298, 2006.

[8] W. Ceusters, P. Martens, C. Dhaen, B. Terzic, "LinkFactory: an advanced formal ontology management System", Proceedings of Interactive Tools for Knowledge Capture (KCAP 2001).

[9] A. Burgun, "Desiderata for domain reference ontologies in biomedicine", Journal of Biomedical Informatics, vol.39, pp.307-313, 2006.

[10] J. Kim, J. Park, "BioIE: Retargetable information extraction and ontological annotation of biological interactions from the literature", Journal of Bioinformatics and Computational Biology, vol. 2, no. 3, pp. 551-568, 2004.

[11] M. Hadzic, E. Chang, "Ontology-based Support for Human Disease Study", Proceedings of the Hawaii International Conference on System Sciences (HICSS38 2005), pp.143a, 2005.

[12] A. S. Sidhu, T. S. Dillon, E. Chang, "Integration of Protein Data Sources through PO", Proceedings of the 17th International Conference on Database and Expert Systems Applications (DEXA 2006), Poland, pp. 519-527, 2006

[13] J. Liu, S.H. Juo, A. Dewan, A. Grunn, X. Tong, M. Brito, N. Park, J.E. Loth, K. Kanyas, B. Lerer, J. Endicott, G. Penchaszadeh, J.A. Knowles, J. Ott, T.C. Gilliam, M. Baron, "Evidence for a putative bipolar disorder locus on 2p13-16 and other potential loci on 4q31, 7q34, 8q13, 9q31, 10q21-24, 13q32, 14q21 and 17q11-12”, Molecular Psychiatry, vol. 8 , no. 3 , pp. 333-342, 2003.

[14] N. Craddock, I. Jones, "Molecular genetics of bipolar disorder", The British Journal of Psychiatry, vol. 178, no. 41, pp. 128-133, 2001.

[15] M. Horvitz-Lennon, A.M. Kilbourne, H.A. Pincus, "From Silos To Bridges: Meeting The General Health Care Needs Of Adults With Severe Mental Illnesses", Health Affairs, vol. 25, no. 3, pp. 659-669, 2006.

[16] D.G. Smith, S. Ebrahim, S. Lewis, A.L. Hansell, L.J. Palmer, P.R. Burton, "Genetic epidemiology and public health: hope, hype, and future prospects", The Lancet, vol. 366, no. 9495, pp. 1484-1498, 2005.

[17] M. Wenner, "Infected with Insanity: Could Microbes Cause Mental Illness?", Scientific American, 2008. Retrieved from http://www.sciam.com/article.cfm?id=infected-with-insanity.

[18]. M. Hadzic, E. Chang, 'Role of the Ontologies in the Context of Grid Computing and Application for the Human Disease Studies', Proceedings of the First International IFIP Conference on Semantics of a Networked World-Semantics for Grid Databases (ACM SIGMOD/POD conference: ICSNW 2004), France, Lecture Notes in Computer Sciences 3226, pp. 316-318, 2004.

[19] C. Wu, M. Hadzic, "Creating Interoperability Within Healthcare Industry", Proceedings of the $6^{\text {th }}$ IEEE International Conference on Industrial Informatics, Korea, 2008.

[20] M. Hadzic, E. Chang, "Ontology-based Multi-agent systems support human disease study and control", in Frontiers in Artificial Intelligence and Applications (special issues on Self-organization and Autonomic Informatics), H. Czap, R. Unland, C. Branki, H. Tianfield, Eds. IOS Press, 2005, pp. 129-141.

[21] M. Hadzic, F. Hadzic, T. Dillon, "Tree Mining in Mental Health Domain", Proceedings of the Hawaii International Conference on System Sciences (HICSS-41), USA, 2008.

[22] M. Hadzic, F. Hadzic, T. Dillon, "Domain Driven Data Mining for the Mental Health Domain", in Domain Driven Data Mining: Domain Problems, P.S. Yu, C. Zhang, H. Zhang, Eds. Springer, 2008.

[23] World Health Organization, "International Statistical Classification of Diseases and Related Health Problems, 10th Revision", World Health Organization, 2007.

[24] American Psychiatric Association, "Diagnostic and Statistical Manual of Mental Disorders, Fourth Edition, Text Revision (DSM-IV-TR)", American Psychiatric Publishing, 2000.
[24] K.S. Kendler and L. Karowski, "Stressful life events and liability to major depression: genetic control of exposure to the environment" Psychol Med, vol. 27, pp. 539-547, 1997.

[25] D. Bhugra \& A. Munro, "Troublesome Disguises: Underdiagnosed Psychiatric Syndromes", Blackwell Science Ltd, 1997.

[26] H. Dilling, "ICD-10 chapter V (F): difficulties and deficiencies" European Psychiatry, vol. 11, no. 4, pp. 177s-178s, 1996.

[27] World Health Organization, "WHO Resource Book on Mental Health: Human rights and legislation" ISBN 924156282 (PDF), 2005.

[28] J.E. Mezzich, "International surveys on the use of ICD-10 and related diagnostic systems" Psychopathology, vol. 35, no. 2-3, pp. 72-5, 2002.

[29] WHO World Mental Health Survey Consortium. Prevalence, severity, and unmet need for treatment of mental disorders in the World Health Organization World Mental Health Surveys. JAMA, vol. 291, no. 21, pp. 2581-90, 2004.

[30] J.J. Schildkraut, "The catecholamine hypothesis of affective disorders: a review of supporting evidence", Am J Psychiatry, vol. 122, no. 5, pp. 509, 1965.

[31] A.F. Oke, R.N. Adams, "Elevated thalamic dopamine: possible link to sensory dysfunctions in schizophrenia", Schizophr Bull, vol. 13, no. 4, pp. 589-604, 1987.

[32] O. Lindsley, B.F. Skinner, H.C. Solomon, "Studies in behavior therapy (Status Report I)". Walthama, MA.: Metropolitan State Hospital, 1953.

[33] D. M. Clark, C. G. Fairburn, "Science and Practice of Cognitive Behaviour Therapy", Oxford University Press, 1997.

[34] A.T. Beck, "Cognitive Therapy and the Emotional Disorders", Intl Universities Press, 1975.

[35] J. Scott, J.M. Williams, A.T. Beck, "Cognitive Therapy in Clinical Practice: An Illustrative Casebook", Routledge, 1989.

[36] C. Aanstoos, I. Serlin, \& T. Greening, "History of Division 32 (Humanistic Psychology) of the American Psychological Association", In D. Dewsbury (Ed.), Unification through Division: Histories of the divisions of the American Psychological Association, Vol. V. Washington, DC: American Psychological Association, 2000.

[37] D. Ray, S. Bratton, T. Rhine, \& L. Jones, "The effectiveness of play therapy: Responding to the critics. International Journal of Play Therapy, vol. 10, no. 1, pp. 85-108, 2001.

[38] F. Leichsenring, "The effectiveness of psychodynamic therapy. A review using criteria of evidence-based medicine", Z Psychosom Med Psychother, vol. 48, no. 2, pp. 139-62, 2002.

[39] C. Reck, C. Mundt, "Psychodynamic therapy approaches in depressive disorders. Pathogenesis models and empirical principles", Nervenarzt, vol. 73, no. 7, pp. 613-9, 2002.

[40] G.L. Klerman, A. Dimascio and M. Weissman, "Treatment of depression by Drugs and Psychotherapy", American Journal of Psychiatry, vol. 131, pp. 186-191, 1974.

[41] A. Saeed, "Integrated Psychiatric Treatment for Mental Disorders", $J R$ Soc Health, vol. 108, no. 3, pp. 107-109, 1988.

[42] T.E. Goldberg, R.E. Straub, J.H. Callicott, A.Hariri, V.S. Mattay, L. Bigelow, R. Coppola, M.F. Egan, D.R Weinberger, "The G72/G30 Gene Complex and Cognitive Abnormalities in Schizophrenia", Neuropsychopharmacology, vol. 31, pp. 2022-2032, 2006.

[43] J. Tsevat, J.G. Solzan, K.M. Kuntz, J. Ragland, J.S. Currier, R.L. Sell, M.C. Weinstein, "Health Values of Patients Infected with Human Immunodeficiency Virus: Relationship to Mental Health and Physical Functioning", Medical Care, vol. 34, no. 1, pp. 44-57, 1996.

[44] S, Ettner, "New evidence on the relationship between income and health", Journal of Health Economics, vol. 15, no. 1, pp. 67-85, 1996.

[45] A.E. Bergin, "Values and Religious Issues in Psychotherapy and Mental Health", American Psychologist, vol. 46, no. 4, pp. 394-403, 1991.

[46] R. D'Souza, 'Do patients expect psychiatrists to be interested in spiritual issues?", Australasian Psychiatry, vol. 10, no. 1, pp. 44-47, 2002.

[47] R. D'Souza, “Incorporating a spiritual history into a psychiatric assessment', Australasian Psychiatry, vol. 11, no. 1, pp. 12-15, 2003.

[48] D. Colbert, "Deadly Emotions: Understand the Mind-Body-Spirit Connection That Can Heal or Destroy You", Thomas Nelson Publishers, 2006.

[49] K.K. Patel, B. Butler, K.B. Wells, "What Is Necessary To Transform The Quality Of Mental Health Care", Health Affairs, vol. 25, no. 3, pp. 681-693, 2006. 